\title{
New polymorphs of isoniazid: discovery from melt experiments
}

\author{
Keke Zhang, ${ }^{\text {a,b }}$ Chunhua Hu, ${ }^{a}$ Alexander G. Shukenberg, ${ }^{a}$ Noalle Fellah, ${ }^{a}$ \\ Michael D. Ward ${ }^{\mathrm{a}, *}$ \\ ${ }^{a}$ Department of Chemistry and Molecular Design Institute, New York University, New York City, \\ NY, 10003, USA \\ b School of Chemical Engineering and Technology, State Key Laboratory of Chemical \\ Engineering, Tianjin University, Tianjin 300072, People's Republic of China.

\section{Email Contact: kz1225@nyu.edu}

Tuberculosis (TB) is a disease that has thousands of years history. ${ }^{1}$ This epidemic disease caused by bacillus Mycobacterium tuberculosis used to be fatal until effective chemotherapy appeared in the 1940s. ${ }^{2}$ For now, there are 20 TB drugs in clinical trials and a lot of efforts are invested into the research about prevention and treatment in TB. Yet, the "end" of TB as major health problem is still unreachable. According to the Global Tuberculosis Report 2018, "TB is one of the top 10 causes of death". ${ }^{3}$ Intensified research about known anti-tubercular drugs and effort to design new drugs are still in urgently demand. Isoniazid (pyrazine-2-carboxyamide, isonicotinic acid hydrazide, INZ, Scheme 1), along with rifampicin, ethambutol, and pyrazinamide was recommended as first-line drug as a 6-month regimen. ${ }^{3}$ INZ is a well-studied pharmaceutical not only used as anti-tuberculosis drugs but also applied as a conformer for codrugs. ${ }^{4,} \mathrm{INZ}$ has a long research history since it was firstly used as an antibiotic in $1952 .{ }^{6}$ In addition to studies of INZ in curing TB mechanisms, there have abundant researches about cocrystals, morphology and solubility of INZ. ${ }^{7,8,9}$ There are four crystal structures of INZ in CSD database, all of them correspond to one polymorph. ${ }^{10,11}$ Here we report discovery of two new polymorphs of INZ by crystallization from the melt, a simple and inexpensive method that we recently applied to discover and characterize new polymorphs of several common and well characterized materials such as coumarin, ${ }^{12}$ aspirin ${ }^{13}$ and malic acid. ${ }^{14}$ It's inspiring that two new polymorphs of INZ were discovered after more than 65 years' bench work for the first time.

\section{Reference}

[1] I. Hershkovitz, H. D. Donoghue, D. E. Minnikin, H. May, O. Y. Lee, M. Feldman, Tuberculosis origin: the Neolithic scenario. Tuberculosis (Edinb). 2015, 95 Suppl 1: S122-6

[2] J. F. Murray, D. E. Schraufnagel, P. C. Hopewell, Treatment of Tuberculosis: A Historical Perspective. Ann Am Thorac Soc, 2015, 12, 1749-1759.

[3] World Health Organization. (2018). Global tuberculosis report 2018. World Health Organization.

[4] A. Lemmerer, J. Bernstein, V. Kahlenberg, One-pot covalent and supramolecular synthesis of pharmaceutical co-crystals using the API isoniazid: a potential supramolecular reagent. CrystEngComm, 2010, 12, 2856-2864.

[5] A. Lemmerer, J. Bernstein, V. Kahlenberg, Hydrogen Bonding Patterns of the Co-Crystal Containing the Pharmaceutically Active Ingredient Isoniazid and Terephthalic Acid. J Chem Crystallogr, 2011, 41, 991-997.

[6] C. Vilchèze, W. R. Jacobs, Jr, The Mechanism of Isoniazid Killing: Clarity Through the Scope of Genetics. Annu. Rev. Microbiol., 2007, 61, 35-50.

[7] D. Han, T. Karmakar, Z. Bjelobrk, J. Gong, M. Parrinello, Solvent-mediated morphology selection of the active pharmaceutical ingredient isoniazid: Experimental and simulation studies. 
Chem. Eng. Sci., 2018, https://doi.org/10.1016/j.ces.2018.10.022.

[8] T. Gong, D. Han, Y. Chen, S. Wang, W. Tang, Solubility and Data Correlation of Isoniazid in Different Pure and Binary Mixed Solvent Systems from 283.15 K to 323.15 K. J. Chem. Eng. Data, 2018, 63(12), 4767-4778.

[9] R. Heryanto, E. C. Abdullah, M. Hasan, Solubility of Isoniazid in Supercritical Carbon Dioxide. J. Chem. Eng. Data, 2010, 55(6), 2306-2309.

[10] L. H. Jensen, The Crystal Structure of Isonicotinic Acid Hydrazide. J. Am. Chem. Soc., 1954, 76, 4463-4467.

[11] G. Rajalakshmi, V. R. Hathwar, P. Kumaradhas, Topological analysis of electron density and the electrostatic properties of isoniazid: an experimental and theoretical study. Acta Cryst. 2014, B70, 331-341.

[12] A. G. Shtukenberg, Q, Zhu, D. J. Carter, L. Vogt, J. Hoja, E. Schneider, H. Song, B. Pokroy, I. Polishchuk, A. Tkatchenko, A. R. Oganov, A. L. Rohl, M. E. Tuckerman, B. Kahr, Powder diffraction and crystal structure prediction identify four new coumarin polymorphs. Chem. Sci., 2017,8, 4926-4940.

[13] A. G. Shtukenberg, C. T. Hu, Q. Zhu, M. U. Schmidt, W. Xu, M. Tan, B. Kahr, The Third Ambient Aspirin Polymorph. Cryst. Growth Des. 2017, 17, 3562-3566.

[14] J. Yang, C. T. Hu, A. G. Shtukenberg, Q. Yin, B. Kahr, L-Malic acid crystallization: polymorphism, semispherulites, twisting, and polarity. CrystEngComm, 2018, 20, 1383.

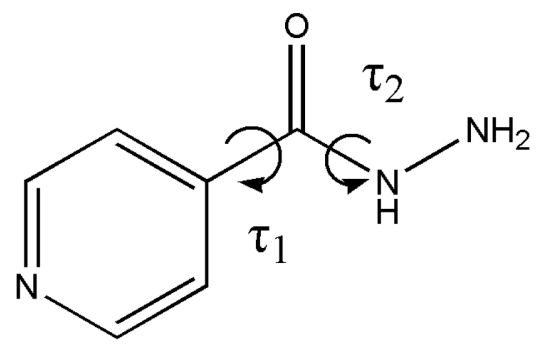

Scheme 1. Molecule structure of isoniazid. 